\title{
Comparative studies on the biochemical composition and polypeptide profiles of the cyst walls from sterile and fertile hydatid cysts of Echinococcus granulosus from buffalo host
}

\author{
M. IRSHADULLAH ${ }^{*}$, M. RANI \\ Section of Parasitology, Department of Zoology, Aligarh Muslim University Aligarh, 202002 India, \\ *E-mail:malikirshadullah@rediffmail.com,malikirshadullah@yahoo.co.in
}

\begin{abstract}
Summary
In the present study, biochemical composition and polypeptide profiles of the cyst walls of sterile and fertile hydatid cyst, collected from the lungs of naturally infected Bubalus bubalis have been investigated. Significant quantitative differences in various biochemical components as well as in protein banding patterns were observed between the two types of cyst walls, suggesting variations in their metabolic states. The cyst wall collected from fertile cysts had significantly less glycogen, lipids and triglycerides but more RNA and DNA as compared to sterile cyst wall. Among phospholipid fractions, lysophosphatidylethanolamine and phosphatidylethanolamine were significantly higher in the fertile, whereas, sphingomyelin showed higher values in the sterile cyst wall. The numbers of polypeptides resolved in the fertile cyst wall were more than those in sterile cyst wall possibly due to the presence of extra proteins required for production of brood capsules and protoscoleces. Detection of more polypeptides by silver than CBBR-250 staining could be due to high sensitivity of silver stain which also detects conjugated proteins such as lipo-, phospho- and glycoproteins. The characteristic polypeptides specific to either types of the cyst wall have the potential to be exploited for diagnostics.
\end{abstract}

Keywords: Biochemical composition; cyst wall; SDSgradient PAGE; polypeptides

\section{Introduction}

Cystic hydatid disease caused by the larval stage of a teaniid cestode Echinococcus granulosus, is one of the most important cosmopolitan parasitic zoonoses. The cyclic transmission of the disease mainly occurs between dog as definitive host and livestock as intermediate hosts. Humans are accidental intermediate host who do not play a role in the natural cycle and are considered as aberrant host. Within intermediate host, onchospheres liberated from ingested viable eggs may develop into hydatid cysts (metacestode larvae) in any organ but liver and lungs are the most preferred sites for cyst development (Turcekova et al., 2009). The developing cyst induces pressure and space occupying effect on different vital organs causing pain, degenerative changes in the infected organs and high economic losses due to condemnation of affected viscera at the slaughterhouses (Gholami et al., 2010). The fully developed mrtacestode of E. granulosus is unilocular, sub spherical, fluid -filled and exhibits least complex structure than other Echinococcus species (Thompson, 1995). The cyst wall consists of an inner thin nucleated germinal layer, which gives rise brood capsules and protoscoleces by budding and an outer thick acellular laminated layer, adjacent to the host-produced adventitial layer. The laminated layer is unique to the genus Echinococcus and is involved in the survival of metacestodes by offering an immunologically inert barrier and denies the access of host defense cells (Coltorti \& Varela-Diaz, 1974; Leducq \& Gabrion, 1992; Taherkhani \& Rogan, 2000). Based on the presence and absence of protoscoleces, the cysts have been classified as sterile (without protoscoleces) and fertile (with protoscoleces). The fertile cysts are responsible for maintaining the life cycle, whereas, morbidity and pathological manifestations occur with all types of cysts.

The available literatures revealed that the biochemical composition of protoscoleces and hydatid fluids from different hosts and habitats have been well studied but only limited work carried out so for on the cyst wall (see references in McManus \& Bryant, 1995). Previous studies carried out on the biochemical composition of the cyst wall from India, were from the cattle and human hosts (Sadanand, 1971; Irshadullah \& Nizami, 1997a), whereas no information is available from buffalo origin which is important from the strain point of view, as E. granulosus exist as different strains and each strain varies in infectivity, biochemical composition, protein profile, sensitivity 
to chemotherapeutic agent and pathology (Thompson \& McManus, 2002). Furthermore, no study has yet been carried out on polypeptide profiles of the cyst wall. A comparative biochemical analysis was therefore undertaken to assess the similarity and/or differences in the organic constituents between the cyst walls of sterile and fertile hydatid cyst of E. granulosus from Indian buffalo, which could be useful for the identification of dominant antigen and could be used for diagnostic purpose.

\section{Materials and methods}

\section{Collection of samples}

Hydatid cysts were collected from the lungs of naturally infected, freshly slaughtered Indian water buffalo Bubalus bubalis (12 to 14 years of age), at the Aligarh abattoir and brought to the laboratory. The cyst walls from the sterile and fertile hydatid cysts were isolated by the methods as described by Irshadullah and Rani (2010) and from here onwards they will be referred as sterile and fertile cyst wall respectively. After isolation, the specimens were thoroughly washed with Hank's medium to remove the protoscoleces and were used for the extraction and estimation of various biochemical components and analysis of polypeptide profile. In total 3 sterile and 3 fertile cyst walls were used.

\section{Biochemical Composition}

Biochemical components were analyzed by using standard spectrophotometric methods and all readings were taken on Spectronic 1001 Spectrophotometer (Bausch and Lomb). Methods followed for the extraction and estimation of alkali soluble glycogen, proteins, lipids, RNA, DNA, and major lipid fractions like cholesterol, triglycerides, free fatty acids and phospholipids were essentially the same as described in Irshadullah and Nizami (1997b). Phospholipids were further fractionated by ascending thin layer chromatography (Skipski et al., 1964) using chloroform : methanol : water $(65: 25: 4, \mathrm{v} / \mathrm{v})$ as the solvent system. The phospholipid fractions were identified after comparing their Rf values with the standard phospholipids (lysophosphatidylcholine, lysophosphatidylethanolamine, phosphatidylcholine, sphingomyelin and phosphatidylethanolamine) (V. P. Chest Institute, New Delhi), applied on the same plate and the phosphorous was analyzed according to the method of Rouser et al. (1970). Student's t-test was employed to detect the level of significance.

\section{Polyacrylamide gel electrophoresis}

Sodium-dodecyl sulphate gradient polyacrylamide gel electrophoresis (SDS-gradient PAGE) was performed according to the method of Laemmli (1970) with minor modification, in a separating $8-15 \%$ gradient slab gel using a vertical gel electrophoresis unit (Atto, Japan). For this study, the sterile and fertile hydatid cyst walls were separately homogenized in $0.1 \mathrm{M}$ phosphate buffer $(\mathrm{pH}$ 7.4), containing 0.25 M sucrose in a Potter Elvehjm glass tissue homogenizer at $4{ }^{\circ} \mathrm{C}$. The soluble protein samples were isolated after centrifugation of homogenates at $1,000 \times \mathrm{g}$ for $20 \mathrm{~min}$ and used for electrophoresis. The protein concentration in the samples was determined by the dye binding method (Spector, 1978), using bovine serum albumin (BSA) as standard. The soluble protein samples as well as standard molecular weight markers (Fermentos, Cat. No SM 0431) were boiled for $5 \mathrm{~min}$ in Laemmli's sample buffer containing $50 \%$ stacking gel buffer $(\mathrm{pH}$, 6.8), $20 \%$ glycerol, $10 \%$ SDS, $20 \% \beta$-Mercaptoethanol and $0.05 \%(\mathrm{w} / \mathrm{v})$ aqueous bromophenol blue as the marker dye. Approximately, $60 \mu \mathrm{g}$ protein samples and $8 \mu \mathrm{g}$ standard protein marker containing, $\beta$-galactosidase $(116 \mathrm{KDa})$, BSA (66.2 KDa), ovalbumin $(45 \mathrm{KDa})$, lactate dehydrogenase $(35 \mathrm{KDa})$, restriction endonuclease Bsp 981 (25 $\mathrm{KDa}), \beta$-lactoglobin (18.4 KDa) and lysozyme (14.4 KDa) were separately loaded onto different lanes of the gel with the help of micro sample applicator. The electrophoresis was carried out at $20 \mathrm{~mA}$ per slab gel until the bromophenol blue tracking dye reached $0.5 \mathrm{~cm}$ above the end of the gel.

After electrophoresis, the gels were fixed in methanol, acetic acid and double distilled water mixture (45:10:45, $\mathrm{v} / \mathrm{v}$ ) for $1 \mathrm{~h}$ at room temperature and stained in $0.25 \%$ (w/v) Coomassie Brilliant Blue R- 250 (CBBR-250) dye (Sigma Chemical Co., USA). Silver staining of the gels was performed according to the method of Sørensen et al. (2002). The gels were fixed with $12 \%(\mathrm{w} / \mathrm{v}) \mathrm{TCA}$ in $50 \%$ $(\mathrm{v} / \mathrm{v})$ methanol for $30 \mathrm{~min}$ and then transferred into ethanol, acetic acid and double distilled water $(10: 5: 85, \mathrm{v} / \mathrm{v})$ mixture followed by $0.057 \%$ ammonium per sulphate solution for $20 \mathrm{~min}$ in each. After thorough washing with double distilled water for $45 \mathrm{~min}$, the gels were transferred into $0.1 \%(\mathrm{w} / \mathrm{v}) \mathrm{AgNO}_{3}$ for one hour. After quick rinse with double distilled water, the gels were placed in freshly prepared developer solution containing $2 \%(\mathrm{w} / \mathrm{v}) \mathrm{Na}_{2} \mathrm{CO}_{3}$ and $50 \mu \mathrm{l}$ of $40 \%$ formaldehyde until bands became clearly visible and the reaction was stopped with $1 \%(\mathrm{v} / \mathrm{v})$ acetic acid. The molecular weights of resolved polypeptides were determined with the help of semi log standard graph plotted by using molecular weight of standard marker proteins against their Rf values.

Table 1. Comparative data on the biochemical constituents of the sterile and fertile hydatid cyst walls isolated from buffalo lungs' cysts

\begin{tabular}{cccc}
\hline $\begin{array}{c}\text { Biochemical } \\
\text { components }\end{array}$ & $\begin{array}{c}\text { Sterile cyst } \\
\text { wall }\end{array}$ & $\begin{array}{c}\text { Fertile cyst } \\
\text { wall }\end{array}$ & p value \\
\hline Glycogen & $0.166 \pm 0.017$ & $0.082 \pm 0.014$ & $p<0.01$ \\
Protein & $60.040 \pm 3.190$ & $58.185 \pm 3.895$ & $p<0.1$ \\
Lipids & $0.911 \pm 0.120$ & $0.655 \pm 0.100$ & $p<0.05$ \\
RNA & $1.167 \pm 0.140$ & $1.472 \pm 0.160$ & $p<0.05$ \\
DNA & $0.081 \pm 0.008$ & $0.182 \pm 0.020$ & $p<0.05$
\end{tabular}

Values are expressed as $\mathrm{mg} / \mathrm{g}$ wet weight of tissue \pm SEM of 3 samples, $p<0.05$ is considered as significant. 


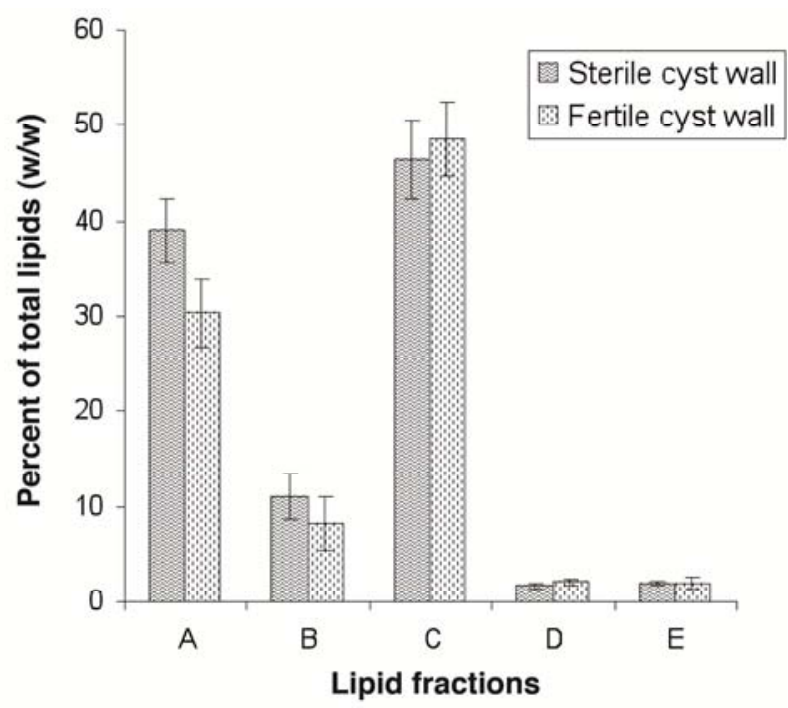

Fig.1. Lipid fractions of the sterile and fertile hydatid cyst wall from buffalo. A - Triglycerides, B - Cholesterol, C - Phospholipid, D - Free fatty acid, E - Unidentified fractions

\section{Results}

The sterile and fertile cyst walls collected from the same host and habitat showed considerable heterogeneity in their organic constituents. The fertile cyst wall had significantly less glycogen $(p<0.01)$, and lipids $(p<0.05)$ whereas more RNA $(\mathrm{p}<0.05)$ and DNA $(\mathrm{p}<0.05)$ than the sterile cyst wall (Table 1). Among known lipid fractions, phospholipids and free fatty acids were higher in the fertile cyst wall, whereas triglycerides and cholesterol in the sterile cyst wall, but only the differences in the triglycerides level were found significant $(\mathrm{p}<0.05)$. The level of these fractions was in the following order: phospholipids $>$ triglycerides $>$ cholesterol $>$ free fatty acids in both types of cyst membrane (Fig. 1). Phospholipids alone constituted about $46.39 \%$ and $48.59 \%$ of the total lipids in the sterile and

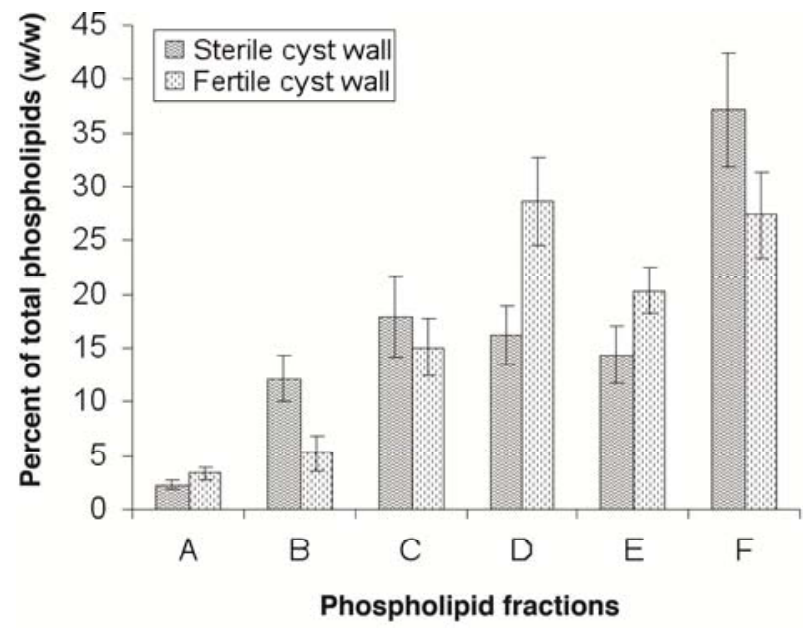

Fig. 2. Phospholipid fractions of the sterile and fertile hydatid cyst walls from buffalo. A - Lysophosphatidylcholine, B - Sphingomyelin, C - Phosphatidylcholine,

D - Lysophosphatidylethanolamine, E - Phosphatidylethanolamine, F - Unidentified fraction fertile cyst wall respectively. Further analysis of phospholipid fractions (Fig. 2) revealed that lysophosphatidylcholine $(\mathrm{p}<1.0)$, lysophosphatidylethanolamine $(\mathrm{p}<$ $0.05)$, and phosphatidylethanolamine $(\mathrm{p}<0.05)$ were higher in the fertile cyst wall, whereas sphingomyelin $(\mathrm{p}<$ $0.02)$ and phosphatidylcholine $(p<1.0)$ in the sterile cyst wall. The unidentified phospholipid fraction constituted $37.15 \%$ and $27.38 \%$ of the total phospholipids in the sterile and fertile cyst wall respectively.

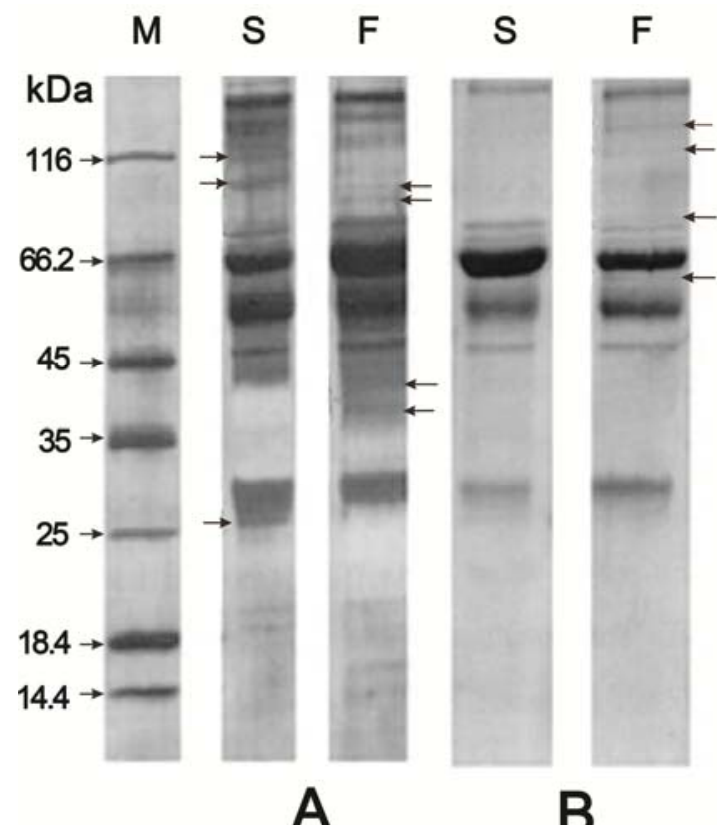

Fig. 3. The polypeptide profiles of sterile (S) and fertile (F) cyst walls along with standard marker proteins (M) of known molecular weight $(\mathrm{KDa})$ by SDS-gradient PAGE. The arrows indicate the characteristic polypeptides in the Silver (A) and Coomassie brilliant blue R - 250 (B) stained gels.

Besides biochemical components, differences were also noticed in the polypeptide profiles between the sterile and fertile cyst wall. A total of 7 and 11 polypeptides by CBBR-250 staining while 21 and 23 polypeptides by silver staining were resolved in the sterile and fertile cyst wall respectively (Fig. 3, A and B). Besides common polypeptides some characteristic polypeptides with different molecular weights were also detected in both the sterile and fertile cyst walls (Table 2).

\section{Discussion}

Among the analyzed organic constituents, protein was found to be the major components in both types of cyst wall, which is in agreement with those reported by Irshadullah and Nizami (1997a) and Sadanand (1971) for the hydatid cyst wall and laminated membrane of E. granulosus from human and cattle hosts respectively. High concentration of protein and lipids in the cyst wall may be due to their requirements for molecular reorganization and membrane synthesis.

Quantitative variations in the biochemical components 
Table 2. Characteristic polypeptides and their molecular weights

\begin{tabular}{|c|c|c|c|c|c|c|c|}
\hline \multicolumn{4}{|c|}{ SILVER STAINED GEL } & \multicolumn{4}{|c|}{ CBBR-250 STAINED GEL } \\
\hline \multicolumn{2}{|r|}{ Sterile cyst wall } & \multicolumn{2}{|r|}{ Fertile cyst wall } & \multicolumn{2}{|c|}{ Sterile cyst wall } & \multicolumn{2}{|c|}{ Fertile cyst wall } \\
\hline No. & $\begin{array}{c}\text { Molecular weight } \\
(\mathrm{KDa})\end{array}$ & No. & $\begin{array}{c}\text { Molecular weight } \\
(\mathrm{KDa})\end{array}$ & No. & $\begin{array}{c}\text { Molecular weight } \\
(\mathrm{KDa})\end{array}$ & No. & $\begin{array}{l}\text { Molecular weight } \\
(\mathrm{KDa})\end{array}$ \\
\hline 1 & 116 & 1 & 99 & Nil & Nil & 2 & $>116$ \\
\hline 1 & 100 & 1 & 92.5 & & & 1 & 80 \\
\hline \multirow[t]{2}{*}{1} & 25.5 & 1 & 37 & & & 1 & 63 \\
\hline & & 1 & 35.5 & & & & \\
\hline
\end{tabular}

between the sterile and fertile cyst wall may indicate variations in their metabolic state as suggested for T. hydatigena cysticerci (Abidi et al., 1989). Comparatively low glycogen level in the fertile cyst wall suggests that this component is being utilized for the formation of brood capsules and protoscoleces, which finds support from the earlier report of the decrease in sugar content in the fibrilar matrix of laminated layer during differentiation of protoscoleces (Leducq \& Gabrion, 1992). Similarly weak reaction of glycogen has been demonstrated histochemically in the fertile than the sterile cyst wall (Irshadullah \& Rani, 2010).

Presence of both DNA and RNA in the hydatid cyst walls understudy may be due to the fact that these components are solely present in the germinal rather than laminated layer, as the germinal membrane contains several cell types and nuclei (Kilejian et al., 1961), while the laminated layer is a polysaccharide protein complex (Kilejian \& Schwabe, 1971). Occurrence of more DNA and RNA in the fertile cyst wall suggests that its germinal layer is more developed than the sterile cyst probably for the production of brood capsules and protoscoleces. This may also reflect increased cellular proliferation taking place in the fertile cysts which affects growth of the cysts, since the fertile cysts grow faster than the sterile cysts (Turcekova et al., 2009). Furthermore, differences in the RNA level indicated variation in the nature and metabolic state of the cyst walls as RNA constitutes a marker for overall metabolic activities (Smith \& Walker, 1986).

Quantitatively lesser amounts of lipids and triglycerides in the fertile cyst wall indicated their utilization for the formation and insertion of brood capsules and protoscoleces since it has been suggested that the resultant products of lipid metabolism are important for the development and growth of protoscoleces (Vercelli-Retta et al., 1975). The lipid and phospholipid fractions obtained in the present study showed similarity as well as differences with the findings of Richards et al. (1987). They detected di and triacylglycerols, cholesterol, wax, steryl esters, oleic acid, sphingomyelin, phosphatidylcholine, phosphatidylinositol and ceramide hexosides in the laminated layer of horse liver hydatid cysts, whereas all components except cholesterol, wax, steryl ester and triacylglycerol in lungs' cysts. Like other cestodes, Echinococcus is not able to synthesize most of their own lipids de novo and therefore, the lipids from the host origin were taken up by the para- site for their survival (Smyth \& McManus, 1989). Furthermore, the phospholipids of host origin in Hymenilepis diminuta (Ginger \& Fairbairn, 1966) and the lack of de novo synthesis of cholesterol in larval cestodes (Frayha, 1968, 1974), suggests that these fractions in the cyst wall may also be of host origin. The high level of phospholipids in both types of cyst walls indicated their possible involvement in the reorganization and synthesis of membrane during growth of the cysts The differential distribution of phospholipid fractions, lysophosphatidylethanolamine and phosphatidylethanolamine in the fertile cyst wall as observed in the present study have also been recorded in higher concentration in other helminthes (Barrett, 1981, Abidi et al., 1989), whereas sphingomyelin and phosphatidylcholine were higher in the sterile cyst wall. The pronounced variation in the unknown phospholipid fractions also suggests that the nature of phospholipids in the two membranes varies considerably. The unknown fraction of phospholipids constituted maximum value. Future studies are, therefore, warranted to identify this fraction by using additional standard phospholipids other than those used in the present study.

Besides biochemical differences, the sterile and fertile hydatid cyst wall also differ in their polypeptide profiles as detected by CBBR-250 and silver staining. An interesting observation was the presence of additional polypeptides in the fertile cyst wall which could be due to the presence/synthesis of additional proteins, required for the production of brood capsules and protoscoleces. Ingold et al., (1998) also found differences in the protein banding pattern between the cysts walls of in vitro cultivated metacestode of E. vogeli and E. multilocularis. Qualitatively different polypeptides can be detected by CBBR-250 (Irie et al., 1982) and silver staining (Dzandu et al.,1984), hence both the techniques were employed in the present study. Detection of more polypeptides by silver staining could be due to higher sensitivity as well as staining property of conjugated proteins like lipo-, phospho- and/or glycoprotein. The specific polypeptides in the sterile and fertile cyst walls not only indicated the biochemical variation but also contribute to the possible existence of antigenic variation and hence they could be exploited for diagnostics.

The presence of host proteins including immunoglobulin on the cyst walls cannot be ruled out, as occurrence of hosts' proteins including immunoglobulin have been re- 
ported on the cyst wall (Varela-Diaz \& Coltorti, 1973; Coltorti \& Varela-Diaz, 1974; Ali Khan \& Siboo, 1981; Taherkhani \& Rogan, 2000). Since we have compared the polypeptide profiles between the sterile and fertile hydatid cyst walls collected from the same host (buffalo) and habitat (lungs), the chances for the occurrence of hosts' proteins are equal on both kinds of cyst walls. Therefore, the observed differences between them give a true picture of variations.

It is likely that both kinds of cyst walls may have same amount of biochemical components during the early phase of development. However, during later stages, these components could be utilized as energy source by fertile cyst for the production of brood capsules and protoscoleces resulting in its decrease in the fertile condition of the cysts. Further studies are required on the parasite materials from experimentally infected host to substantiate this assumption.

\section{Acknowledgements}

The authors are grateful to the Chairman, Department of Zoology, Aligarh Muslim University, Aligarh for providing laboratory facilities and to the Dean, Faculty of Life Sciences for financial assistance.

\section{References}

Abidi, S. M. A., Nizami, W. A., Khan, P., Ahmad, M., IRSHADULLAH, M. (1989): Biochemical characterization of Taenia hydatigena cysticerci from goats and pigs. J. Helminthol., 63: 333 - 337

Ali-Khan, Z., SiBOO, R. (1981): Echinococcus multilocularis: Distribution and persistence of specific host immunoglobulins on cyst membranes. Exp. Parasitol., 51: 159 - 168 BARRETT, J. (1981): Biochemistry of parasitic helminthes. Mcmillan Publishers Ltd., London, 307pp.

Coltorti, E. A., VArela-Diaz, V. M. (1974): Echinococcus granulosus: Penetration of macromolecules and their localization on the parasite membranes of cysts. Exp. Parasitol., 35: 225 - 231. DOI: 10.1016/0014-4894(74)90026-5 DzAndu, J. K., Mercy, E. D., Eenise, L. B., Garry, E. W. (1984): Detection of erythrocyte membrane proteins, sialoglycoproteins and lipids in the same polyacrylamide gel using a double staining technique. Proc. Nat. Acad. Sci. U.S.A., 81: $1733-1737$

FRAYHA, G. J. (1968): A study on the synthesis and absorption of cholesterol in hydatid cysts (Echinococcus granulosus). Comp. Biochem. Physiol., 27B: $875-878$

FRAYHA, G. J. (1974): Synthesis of certain cholesterol precursors by hydatid protoscolices of Echinococcus granulosus, E. multilocularis and cysticerci of Taenia hydatigena. Comp. Biochem. Physiol., 49B: 93 - 98

GHolami, S., Irshadullah, M., MobedI, I. (2010): Rostellar hook morphology of larval Echinococcus granulosus isolates from the Indian buffalo and Iranian sheep, cattle amd camel. J. Helminthol., 1 - 7. DOI: 10.1017/S0022149 $\mathrm{X} 10000520$.
GINGER, C. D., FAIRBAIRN, D. (1966): Lipid metabolism in helminth parasites. II. The major origins of the lipids of Hymenolepis diminuta (Cestoda). J. Parasitol., 52: 1097 1107

InGOLD, K., GotTstein, B., HemPhill, A. (1998): Identification of a laminated layer-associated protein in Echinococcus multilocularis metacestodes. Parasitology, 116: $363-372$

Irie, S., Sezaki, M., Kato, Y. (1982): A faithful double stain of proteins in the polyacrylamide gels with coomassie blue and silver. Anal. Biochem., 126: 350 - 354

IRSHADUlLAH, M., NiZAMI, W. A. (1997a): A case study of human hepatic hydatidosis and the biochemical profile of cyst wall and fluid. Ind. J. Commun. Health, 9, 1: 47 - 52 IRShadullah, M., NizAMI, W. A. (1997b): Biochemical characterization of protoscoleces isolated from buffalo hepatic and pulmonary hydatid cyst. J. Parasit. Anim. Biol. $6,1: 13-24$

IRSHADULLAH, M., RANI, M. (2010): Histochemical studies on the metacestode of Echinococcus granulosus from Indian buffalo origin. J. Vet. Parasitol., 24, 1: 33 - 37

Kilejian, A., Schwabe, C. W. (1971): Studies on the polysaccharides of the Echinococcus granulosus cysts, with observations on a possible mechanism of laminated membrane formation. Comp. Biochem. Physiol. 40B: 25 - 36

Kilejian, A., Schinazi, L. A., Schwabe, C. W. (1961): Host-parasite relationship in echinococcosis. V. Histochemical observation on Echinococcus granulosus. J. Parasitol. 47: $181-188$

LAEMMLI, U. K. (1970): Cleavage of structural proteins during the assembly of the head of bacteriophage T4. Nature, 227: 680 - 685. DOI: 10.1038/227680a0

LEDUCQ, R., GABRION, C. (1992): Developmental changes of Echinococcus multilocularis metacestodes revealed by tegumental ultrastructure and lectin-binding sites. Parasitology, 104: 129 - 141

McManus, D. P., BRyant, C. (1995): Biochemistry, physiology and molecular biology of Echinococcus. In: THOMPSON, R. C. A., LYMBERY, A. J. (Eds.) Echinococcus and hydatid disease. CAB International, Wallingford, UK, pp. $135-181$

Richards, K. S., Ilderton, E., Yardley, H. J. (1987): Lipids in the laminated layer of liver, lung and daughter hydatid cysts of equine Echinococcus granulosus (Cestoda). Comp. Biochem. Physiol. 86B: 209 - 212. DOI: 10.1016/0305-0491(87)90199-4.

Rouser, G., Fleischer, S., Yamamoto, A. (1970): Two dimensional thin layer chromatographic separations of polar lipids and determination of phospholipids by phosphorous analysis of spots. Lipids, 5: $494-496$

SADANAND, A. V. (1971): Biochemical analyses of the cyst wall of Echinococcus granulosus Batsch. Comp. Biochem. Physiol. 40B: 797 - 805. DOI: 10.1016/0305-0491 (71)90153-2.

SkiPSKi, V. P., Peterson, R. F, BARClay, M. (1964): Quantitative analysis of phospholipids by thin layer chromatography. Biochem. J., 90: $374-378$ 
Smith, F. F., WALKer, C. W. (1986): Biochemical changes in the composition of the testes during spermatogenesis in the sea star Asterias vulgaris. J. Exp. Zool., 237: 351 364. DOI: $10.1002 /$ jez.1402370308.

SmYth, J. D., Mcmanus, D. P. (1989): The Physiology and Biochemistry of Cestodes. Cambridge University Press, Cambridge, 367pp.

Sørensen, B. K., HøJRUP, P., ØSTERGÅrD, E., JøRGENSEN, C. S., ENGHILD, J., RYDER, L. R., HoueN, G. (2002): Silver staining of proteins on electroblotting membranes and intensification of silver staining of proteins separated by polyacrylamide gel electrophoresis. Anal. Biochem., 304: 33 - 41. DOI: $10.1006 /$ abio.2001.5604

SPECTOR, T. (1978): Refinement of the coomassie blue method of protein quantitation. Anal. Biochem., 86: $142-$ 146. DOI: 10.1016/0003-2697(78)90327-5

TAHERKHANI, H., RogAN, M. T. (2000): General characterization of laminated layer of Echinococcus granulosus. Iran. J. Med. Sci., 25: 95 - 104
Thompson, R. C. A. (1995): Biology and systematics of Echinococcus. In: THOMPSON, R. C. A., LYMBERY, A. J. (Eds.) Echinococcus and hydatid disease. CAB International, Wallingford, UK, pp. $1-50$

Thompson, R. C. A., McManus, D. P. (2002): Towards a taxonomic revision of the genus Echinococcus. Trends Parasitol.18: 452 - 457

Turcekova, L., Snabel, V., DudinaK, V., Gaspar, V., DUBINSKY, P. (2009): Prevalence of cystic echinococcosis in pigs from Slovakia, with evaluation of size, fertility and number of hydatid cysts. Helminthologia, 46, 3: $151-158$. DOI: 10.2478/s11687-009-0029-4

VArela-Diaz, V. M., Coltorti, E. A. (1973): The presence of host immunoglobulins in hydatid cyst membranes. J. Parasitol., 59: 484 - 488

VERCELli-Retta, J., Reissenweber, N. J., LoRAnZO, W., SIRI, A. M. (1975): Histochemistry and histoenzymology of the hydatid cyst. (Echinococcus granulosus Batsch 1786). Parasitol. Res., 48: 15 - 23. DOI: 10.1007/BF00389825 